\title{
CD38 and CD157 as Receptors of the Immune System: A Bridge Between Innate and Adaptive Immunity
}

\author{
Fabio Malavasi, ${ }^{1,2}$ Silvia Deaglio, ${ }^{1,2}$ Enza Ferrero, ${ }^{1,2}$, Ada Funaro, ${ }^{1,2}$ Jaime Sancho, ${ }^{3}$ Clara M Ausiello, ${ }^{4}$ \\ Erika Ortolan, ${ }^{1,2}$ Tiziana Vaisitti, ${ }^{1,2}$ Mercedes Zubiaur, ${ }^{3}$ Giorgio Fedele, ${ }^{4}$ Semra Aydin, ${ }^{1}$ Elena V Tibaldi, ${ }^{1,2}$ \\ Ilaria Durelli, ${ }^{2}$ Riccardo Lusso, ${ }^{2}$ Franco Cozno, ${ }^{5}$ and Alberto L Horenstein ${ }^{1,2}$
}

${ }^{1}$ Laboratory of Immunogenetics, Department of Genetics, Biology and Biochemistry, University of Torino Medical School, Torino, Italy; ${ }^{2}$ Research Center on Experimental Medicine (CeRMS), University of Torino Medical School, Torino, Italy;

${ }^{3}$ Departamento de Biología Celular e Inmunología, Instituto de Parasitología y Biomedicina "López-Neyra", CSIC, Armilla, Spain; ${ }^{4}$ Department of Infectious Parasitic and Immune-mediated Diseases, Istituto Superiore di Sanita', Rome, Italy; ${ }^{5} \mathrm{HuGeF}$,

First Surgical Clinics, University of Torino Medical School, Torino, Italy

\begin{abstract}
This paper reviews some of the results and the speculations presented at the Torino CD38 Meeting in June, 2006 and focused on CD38 and CD157 seen as a family of molecules acting as surface receptors of immune cells. This partisan view was adopted in the attempt to combine the enzymatic functions with what the immunologists consider key functions in different cell models. At the moment, it is unclear whether the two functions are correlated, indifferent, or independent. Here we present conclusions inferred exclusively on human cell models, namely T and B lymphocytes, dendritic cells, and granulocytes. As an extra analytical tool, we try to follow in the history of life when the enzymatic and receptorial functions were generated, mixing ontogeny, membrane localization, and cell anchorage.
\end{abstract}

Online address: http://www.molmed.org

doi: 10.2119/2006-00094.Malavasi

\section{INTRODUCTION}

This manuscript focuses on the evolutionary story of CD38 and CD157 from soluble enzyme of sea mollusks to surface receptors of leukocytes, as described in talks presented at the CD38 meeting (June 10-12, Torino, Italy). We will start by summarizing the presentation by E Ferrero on the evolutionary strategies behind the unique developmental pathway followed by the CD38 gene family. Next, we will analyze some of the immunological aspects of the human CD38 and CD157 molecules, highlighting the common threads emerging from the analysis of different lineages and models, such as T cells (J Sancho), B cells (S Deaglio), dendritic cells (CM Ausiello), and granulocytes (A Funaro).

\section{CD38 AND CD157: FROM ENZYMES TO RECEPTORS IN PHYLOGENY}

Leukocyte receptors would seem to have nothing to do with enzymes, classically portrayed as intracellular nanomachines that churn out new products from one substrate or another. For at least one molecule, this neat division of roles came tumbling down thanks to a single protein sequence alignment (1). At the time, CD38 was a T lymphocyte receptor enjoying a distinguished career as a research tool, diagnostic marker, and therapeutic target in blood cancers. The startling result of this alignment was that the receptor CD38 was related to a NADase/ADPR cyclase (NADase/ADPRC) found in the gonads of Aplysia californica, a sea mollusk.

Address correspondence and reprint requests to Fabio Malavasi, Laboratory of Immunogenetics, Dept. of Genetics, Biology and Biochemistry, Via Santena, 19, 10126 TORINO, Italy.e-mail: fabio.malavasi@unito.it

Submitted November 2, 2006; accepted for publication December 7, 2006

The first step toward solving the enigma of CD38 and CD157 featuring simultaneously as ectoenzymes and receptors starts from the study of molecular evolution as a source of potential explanations of biological phenomena. In this case, the question is by what mechanisms did a cellular enzyme expressed in Aplysia ovotestis find its way to the surface of human leukocytes?

NADase/ADPRCs enzymatic activities are found in bacteria $(2,3)$, plants $(4)$, and metazoans (5). The eukaryotic NADase/ ADPRCs form a unique gene family of very small size, which is convenient when trying to catalog new members as orthologs (same gene, different species) or paralogs (genes related by duplication). From sea slug to schistosome, sea urchin, chicken, mouse, macaque, and human, the NADase/ADPRC genes all derive from a common ancestral gene. The oldest well-characterized NADase/ ADPRC gene at the moment belongs to Aplysia, whose origins date back to the Cambrian period over 500 million years ago (mya). The compact 8-exon, $7 \mathrm{~kb}$ 
gene reaches $90 \mathrm{~kb}$ by the time humans evolve, essentially through expansion of the intervening sequences.

Mammals have two NADase/ADPRCs identified as CD38 and CD157 (Figure 1). Their genes are very similar in intronexon structure and reflect their common origins with the Aplysia ADPRC gene. Arranged head-to-tail in tandem on HSA chromosome 4 (telomere $\rightarrow$ CD157 $\rightarrow$ CD38 $\rightarrow$ centromere) and MMU chromosome 5, CD38 and CD157 are clearly derived by gene duplication (6). In trying to estimate the date of the duplication event, we are currently characterizing the NADase/ADPRCs in the chicken, a non-mammalian vertebrate. The presence of both CD38 and CD157 in the chicken genome would indicate that the gene duplication pre-dated the reptile/ mammal separation over 300 mya (E Ferrero, unpublished). Analysis of the chicken genome databases and our unpublished data confirm that orthologs of CD38 and CD157 lie in tandem on GGA chromosome 4 (E Ferrero, unpublished).

The products of the NADase/ADPRC genes are either soluble (aplysia, sea urchin) or membrane-bound enzymes [sea urchin (?), schistosome NACE, CD38 from chicken to human, CD157 from chicken (?) to human]. These proteins range in size from the soluble $29-\mathrm{kDa}$ Aplysia ADPRC to the $45-\mathrm{kDa}$ membrane glycoproteins CD38 and CD157. All these proteins have a polypeptide core of 280 300 amino acids with at least $25 \%$ of their residues in common. Their $3 \mathrm{D}$ structures are spectacularly conserved, to which the preservation of ten cysteine residues and the inter-cysteine spacing contribute enormously $(7,8)$. The enzymatic characteristics of the molecules are analyzed in different sections of this issue.

Aplysia cyclase shares $25-30 \%$ of their amino acid sequence with CD38 and CD157 polypeptides, but it is the $\mathrm{N}$ - and C-terminal modifications which determine protein topology and localization. The short N-terminal hydrophobic tract found in Aplysia cyclase is longer in CD38, a process which simply required the addition of a few amino acid

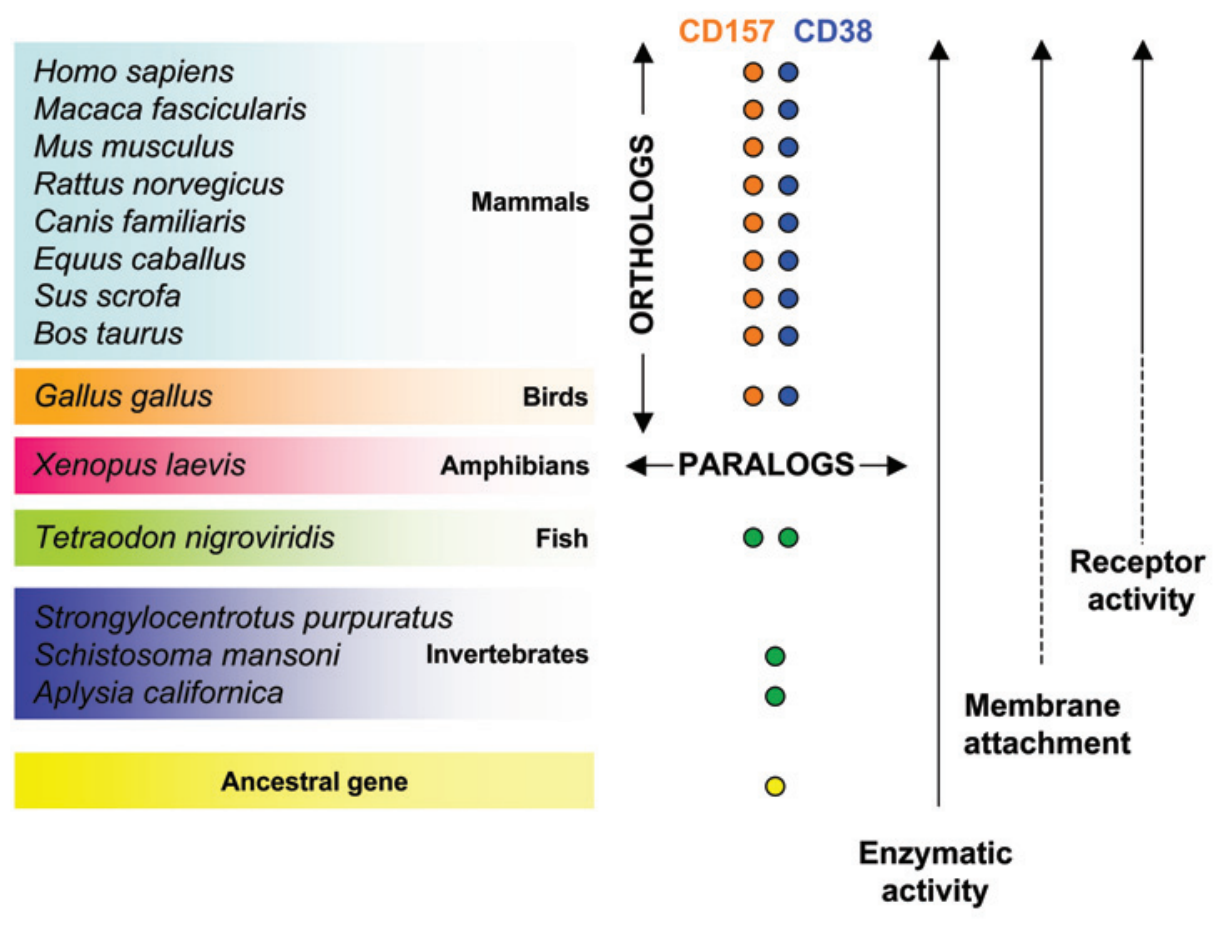

Figure 1. Schematic diagram showing hypothetical steps leading to receptor activity of the ADPRCs. Colored dots represent individual genes; the ancestral gene (yellow dot) is hypothetical.

residues to produce a type II membrane protein with an N-terminal transmembrane domain. The fate of most type II proteins is association with the plasma membrane, Golgi apparatus, and endoplasmic reticulum (ER) (9), and this too was the case of CD38, which thus became an ectoenzyme during its long journey in evolution.

CD157 devised a more innovative mechanism for acquiring membrane attachment. With an N-terminal ERtargeting signal peptide in place, membrane binding was acquired through the addition of a ninth exon whose sole raison d'être is to encode a C-terminal hydrophobic stretch, which is subsequently removed upon addition of glycosyl-phosphatidylinositol (GPI). The process generated a membrane-bound, GPI-anchored NADase/ADPRC.

NADase/ADPRCs inside the cell (including in mitochondria and the nucleus) are predicted to control metabolic pathways and other fundamental cellular processes (10). Confirming this in humans and mice, CD38 has been implicated in insulin secretion $(11,12)$, myometrial contraction (13), airway smooth muscle contractility (14), osteoclast activity (15), and more.

The microevolutionary processes that modified the vertebrate NADase/ ADPRC genes and their effects on protein dynamics provide an explanation for how the vertebrate enzymes reached the cell surface, and the distribution of the metabolic effects within cellular organelles could explain why. A relevant issue is whether the presence of these ectoenzymes on the surface of leukocytes is just an evolutionary trade-off or might it be advantageous to have NADase/ ADPRCs on their surface. Although the plasma concentration of NAD is very low, there might be circumstances in which blood cells are somehow exposed to exceptional amounts of it. Another possibility is that natural selection favored a past organism whose leukocytes 
had at least one NADase/ADPRC on the cell surface, as part of the process of building an enzymatically active membrane surface. No protein acts alone and the NADase/ADPRCs may be part of a cell surface metabolic pathway or network, a notion confirmed by the fact that over $3 \%$ of leukocyte surface molecules are ectoenzymes (16).

It would be nice to find evidence that the first lymphocytes of the vertebrate protoimmune system to emerge in some primitive jawed fish over 500 mya expressed a NADase/ADPRC on their surface. Indeed, the first in silico evidence of a tandem duplication of NADase/ ADPRC genes comes from Tetraodon negroviridis (Figure 1). Once the classical modular antigen receptors of $\mathrm{T}$ and $\mathrm{B}$ (TCR and BCR) lymphocytes appeared, CD38 learned how to parasitize them and their signaling pathways. Human peripheral blood $\mathrm{T}$ and B lymphocytes respond to agonistic anti-CD38 mAbs (in lieu of a presumed more natural ligand) in many ways, including cell activation and proliferation, transcription of cytokine genes, apoptosis, tyrosine phosphorylation of intracellular proteins, and more. CD38 cannot signal alone, its signaling pathways share several steps with those of the TCR and BCR, whose presence is indispensable for CD38 signaling. Apparently enzymatic activities are not involved in the process in human models: hence, by transducing a signal from outside to inside the cell and inducing biological effects, CD38 gains the attribution of a full receptor. Dendritic cells also make use of the signaling capacity of CD38 (vide infra). Instead, cousin CD157 features prominently on the surface of human neutrophils and endothelium (vide infra). In other cells, CD157 performs as receptors do $(17,18)$, leaving little doubt to the dual functional capacity of both CD38 and CD157. To top it all, CD38 and CD157 are very tightly regulated during leukocyte ontogenesis, a process which is energetically quite expensive.

In conclusion, the CD38 and CD157 genes are duplicates and, as such, could have undergone pseudogenization and disappeared without a trace. They have survived with modification to join the ranks of genes pertaining to chemosensation, immunity, and reproduction, those most frequently duplicated during mammalian evolution (see model in Figure 1).

\section{CD38 AS A RECEPTOR IN HUMAN T LYMPHOCYTES}

CD38 functions in T lymphocytes are mediated by cell surface association with the TCR/CD3 complex (19-21): the localization of CD38 to lipid rafts is essential for CD38-mediated signaling in murine $\mathrm{T}$ cell lines transfected with the human CD38 gene (21), or in human Jurkat T line which constitutively expresses CD38 (22). In both models, CD38 appears to localize to the rafts without the need for ligation $(21,22)$. Membrane analysis revealed that CD38, Lck and CD3- $\zeta$ reside in a raft subset different from that hosting LAT and other signaling molecules (22). These results are in line with evidence coming from resting $\mathrm{T}$ lymphocytes, where Lck and LAT are located in different raft subsets (23-25). Cell activation induces the rafts to coalesce, leading Lck and LAT to localize in the same raft subset (23-25). CD38 residency in a subset of rafts together with Lck and CD3- $\zeta$ provides a trigger for CD38-mediated signaling, followed by phosphorylation of $\mathrm{CD} 3-\zeta$, CD3- $\varepsilon$, Lck and LAT. Meanwhile, key signaling molecules (e.g., Sos and p85phosphatidylinositol 3-kinase) are translocated to rafts (22).

The simultaneous presence of antigenloaded HLA Class II molecules, tetraspanins, and CD38 in physically discrete microdomains likely influences the ability of APCs to stimulate antigenspecific T cells. Evidence derived from monocytes, dendritic cells, and Thl response seem to support this view (vide infra).

The mechanisms by which CD38 is included or excluded from rafts in T lymphocytes are not known: a reasonable hypothesis is that the composition of membrane rafts and the dynamics between resting and activated/effector con- ditions may make the difference. What is a firm point is that murine and human $\mathrm{T}$ cell lines with an effector (activated) phenotype express high levels of the ganglioside GM1 (a conventional raft marker) and CD38 is readily detected in rafts $(21,22)$. Resting human $\mathrm{T}$ lymphocytes display CD38 as almost exclusively residing in soluble fractions. The picture is significantly modified after activation (e.g., in vitro PHA), with quantitative and qualitative variations in the distribution of CD38 between raft and non-raft fractions. CD38 modulation correlates with an increased expression of surface GM1 and a distinct expansion of GM1 ${ }^{+}$ CD $38^{+} \mathrm{T}$ lymphocytes (26). Likewise, a significant fraction of CD38 is associated with lipid rafts in Systemic Lupus Erythematosus (SLE) T lymphocytes, which again correlates with increased basal expression of surface GM1. Indeed, SLE $\mathrm{T}$ lymphocytes are reported as displaying basal levels of GM1 higher than normal counterparts: the inference is that they contain similarly high amounts of the raft-fraction-containing CD38 (26). These findings may be indicative of an "activated" phenotype, potentially resulting from the exposure of SLE T lymphocytes to differentiated dendritic cells found in abnormally high numbers in these patients (27).

\section{CD38 AS A RECEPTOR IN HUMAN B LYMPHOCYTES}

Attention to the role of CD38 in human B lymphocyte physiology has recently grown due to a number of reports pointing to a role for CD38 in the pathogenetic network underlying chronic lymphocytic leukemia (CLL) (28). CD38 is a useful negative prognostic marker that correlates with a shorter survival, a more frequent therapeutic need, and a diminished sensitivity to chemotherapeutic agents (reviewed in 29). Our hypothesis is that CD38 is not a simple marker, but that it directly contributes to the worsening of CLL prognosis (30). This was shown in vitro by mimicking the interactions between CLL and nurse-like cells in the blood 
and in peripheral lymphoid organs (31). The results indicate that CD38 ligation induces proliferation and increases survival of a subset of leukemic cells which acquire blastic features together with the surface expression of survival receptors. The interest now is focused on the understanding of the signaling pathway controlled by CD38 in CLL cells and of the functional relationship between this surface receptor and ZAP-70, an intracytoplasmic kinase expressed by a subset of CLL cases and also associated with an unfavorable prognosis. The results obtained in this field are reported elsewhere in this issue.

We are currently expanding these results trying to obtain a general picture of how CD38-mediated signals originate and are transmitted within a B lymphocyte. As described above, experience with $\mathrm{T}$ cells has shown that membrane organization and lateral associations are crucial for CD38-mediated signals. In a recent and unpublished work [presented as a poster at the meeting (Vaisitti et al.)], the localization of CD38 in distinct membrane domains was examined by means of biochemical fractionation of surface membranes. The results indicate that CD38 molecules do not have preferred membrane localization under resting conditions, with approximately one third of the molecular pool present in the detergent-insoluble fractions of the membrane. One third is present in the detergent-soluble fractions while the remaining molecules are visible in the intermediate fractions. These findings suggest that CD38 molecules have a dynamic behavior and membrane relocalization is likely an important functional step in CD38 signal transduction (Figure 2). Further, co-immunoprecipitation experiments have highlighted a physical association between CD38 and the CD19/CD81 complex. This association is also highly dynamic, taking place preferentially within detergent soluble membranes: the findings suggest that it also has a functional nature, in analogy with that described above for T cell models.

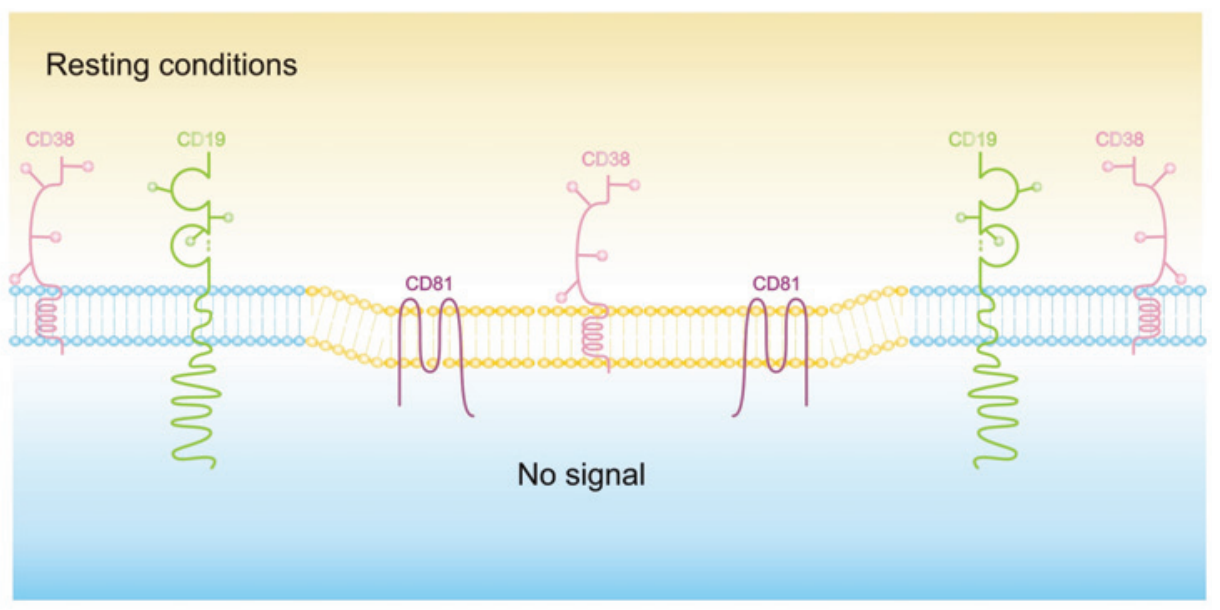

\section{CD31 binding}

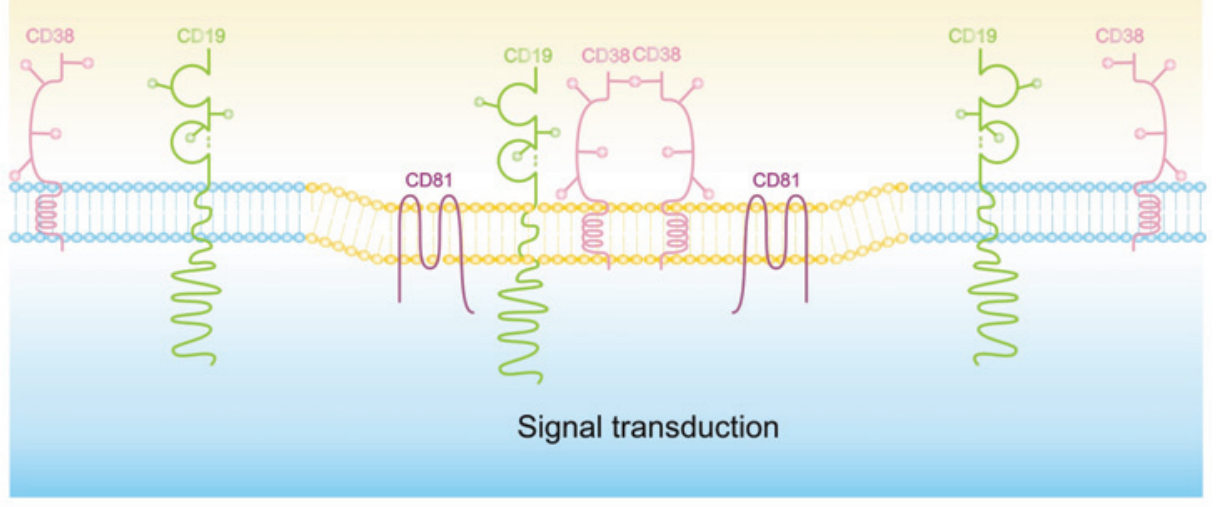

Figure 2. Modulation of CD38 receptor functions in immune cells. Lateral associations with signaling molecules and localization in membrane lipid microdomains are necessary requisites for CD38-mediated signals. B lymphocytes are shown as paradigmatic of other lineages. Lipid rafts are outlined in yellow in the context of the membrane (in blue).

\section{CD38 AS A RECEPTOR IN DENDRITIC CELLS}

Dendritic cells (DC) are called a front line of defense to pathogenetic microorganisms and a reference center in the regulation of quality, quantity, and duration of innate and acquired immune responses (32). When immature, DC capture microbial antigens at the site of infection. Maturation is induced by pathogen recognition receptors (PRR) as well as by an indirect sensing of infections through inflammatory cytokines. Maturation is the result of a rearrangement of genes regulating the production of different cytokines and bringing DC to migrate to lymph nodes, where they present antigens to naive $\mathrm{T}$ cells and polarize the adaptive immune response (32). CD38 is a marker of the transition of monocytes to dendritic cells induced by inflammatory processes (33). The first step to immature DC (iDC) is witnessed by a progressive loss of surface CD38 as well as CD14, and by the simultaneous acquisition of CD1a. Lipopolysaccharide (LPS) induces DC to shift to a mature state. LPS-induced maturation of iDC $\left(\mathrm{CD} 1 \mathrm{a}^{\mathrm{hi}} / \mathrm{CD} 14^{\mathrm{lo}} / \mathrm{CD} 38^{\mathrm{l}}\right)$ is paralleled by a rapid re-expression of $\mathrm{CD} 38$ by the majority of the cells, a process highly reminiscent of lymphocyte differentia- 
tion (34). The role of CD38 in the interplay between DC and effector T lymphocytes was analyzed step by step when DC were directly exposed to pathogens via PRR or indirectly detected inflammatory products.

Direct microbial effects surrogated by LPS induce DC to maturate and express CD38 (along with the reference marker CD83). IFN- $\gamma$ mimicks the effects ruled by the effectors of innate immunity (NK cells and macrophages) and by T lymphocytes, and is generally considered as a broader activation signal operating during DC maturation. DC treated with IFN- $\gamma$ upmodulate CD38 at levels significantly higher than the reference CD83 (33). CD40 ligation (a late T-cellderived signal of DC maturation operating in the secondary lymphoid organs) induces a similar up-regulation of CD38, even if lower to that of CD83. CD38 may be considered an early maturation marker induced by microbial molecules and cytokines produced in early steps in the innate immune response. Late events (for example, driven by CD40 ligation) are less important in the induction of CD38 (33)

These findings support the hypothesis that CD38 may act as a surface molecule modulating adhesion and signaling between DC and T lymphocytes. Owing to its intrinsic ineptitude to act as a receptor, the search was addressed to candidate surface molecules working as transducers. The results indicate that CD83, a marker of human mature DC and a key player in mDC-mediated $\mathrm{T}$ cell activation, is constantly membrane-associated with CD38. Secondly, this association can be instrumental in $\mathrm{mDC}$-mediated $\mathrm{T}$ cell activation of naive T cells $\left(\mathrm{CD} 31^{+}\right)$. Further, other molecules implicated in migration (for example, CD11b and CCR7) are also constantly associated with CD38. On the contrary, no association was found with other signaling receptors, such as CD86 or with CD31. Lastly, the signaling role of CD38 was indirectly confirmed by its localization in lipid rafts, key structures in initiating and sustaining signaling events (35). Immature
DC $\left(C C R 7^{+}\right)$migrate from the skin and mucosal epithelia to the draining lymphoid tissue, where they interact with naive T cells. The maturation is characterized by a progressive loss of the ability to endocytose. The experience gathered on the role of CD38 in rolling and adherence of lymphocytes through interaction with CD31 expressed by endothelial cells was transferred to the analysis of the migration properties of DC.

Chemotaxis and trans-endothelial passage in response to CCL21 indicate that migration of LPS-matured DC is significantly inhibited in the presence of blocking anti-CD38 mAb and of recombinant soluble CD38 (sCD38). Significant inhibitions are also observed in the presence of anti-CD31 mAb. The enzymatic activity of CD38 is not involved in this context, because the addition of 8-Br-cADPR, an antagonist of cADPR, does not influence chemotaxis and trans-endothelial migration. Latest in vitro evidence on human models indicate that the receptorial activity of CD38 is involved in DC migration, even if the contribution of NAD substrate or other end-products (e g., ADPR and NAADP) cannot be excluded (35).

Another issue considered was the analysis of a potential link between CD38 and cellular longevity. Pathogenor $\mathrm{T}$ cell-derived maturation stimuli confer resistance to environmental and intrinsic death signals in DC by regulating the expression of the anti-apoptotic factor Bcl-2 and the pro-apoptotic factor Bax, a mediator of mitochondrial damage. Signals mediated by CD38 converge on these pathways and finely tune the expression of pro- and antiapoptotic molecules, increasing DC longevity, at least for the time needed to reach lymph nodes and prime $\mathrm{T}$ lymphocytes. Inhibition of CD38 engagement during LPS-driven maturation increases the sensitivity of mDC to intrinsic pathways of apoptosis after LPS treatment. LPS-induced survival is reacquired when the signals mediated by CD38 are restored (35).

Another critical point considered was the role of CD38 in the polarization of
$\mathrm{T}$ helper cells (Th)1. The regulation of Th cell development by DC is a complex interplay induced by different pathogenassociated molecules and their interactions with PRR. Protective Th1 responses are directed against intracellular pathogens (e g., virus and intracellular bacteria), while Th2 responses operate against helminths and allergy-induced events. Exacerbated Th1 or Th2 responses are regulated by fine-tuning: for instance, a type of polarized response (i.e., Th17) is specifically involved in autoimmune diseases (36). CD38 is involved in the Th1 response induced by LPS-matured DC. Indeed, inhibition of CD38 signals during the LPS-induced maturation process affects $\mathrm{mDC}$-driven IFN- $\gamma$ production by naive $T$ lymphocytes due to an impaired IL-12p70 secretion by mDC. IL-12 secretion is restored upon CD38 ligation by the agonistic anti-CD38 mAb (35).

\section{CD157 AS A RECEPTOR}

CD157 is a GPI-anchored ectoenzyme showing $36 \%$ amino acid identity with human CD38 and 33\% with the endocellular soluble ADPRC from Aplysia californica (6). Originally identified as a bone marrow (BM) stromal cell molecule (BST-1) (37), human CD157 is prevalently expressed by cells of the myeloid lineage and exerts enzymatic activities along with receptor functions. Early evidence for the receptor nature of CD157 came from the BM microenvironment where it is expressed by stromal cells and supports the growth of pre-B lymphocytes (38). Functional analyses by means of agonistic mAb mimicking the natural ligand(s), suggest that CD157 could act as a receptor with signal transduction capacity. Indeed, CD157 ligation induces tyrosine phosphorylation of a $130 \mathrm{kDa}$ protein identified as focal adhesion kinase (FAK) in selected myeloid cell lines $(39,40)$. Further, it regulates calcium homeostasis and promotes polarization in neutrophils and mediates superoxide $\left(\mathrm{O}_{2}{ }^{-}\right)$production in the human $\mathrm{U} 937$ myeloid line $(41,42)$.

Lacking a cytoplasmic domain, CD157 must associate with some professional 
receptor(s) to transduce signals; much in the same way as CD38, which has a short intracellular domain, parasites specific receptors to compensate for its structural ineptitude to exert receptor functions. Recent results suggest that CD157 associates with the CD11b/CD18 complex for signal transduction on human neutrophils. Indeed, $\beta_{2}$ integrin and CD157 appear to be closely associated either spatially (ligand-induced clustering of $\beta_{2}$ integrin causes colocalization with CD157), or structurally (CD157 co-immunoprecipitates CD18 and vice versa) (42) (and A.F., manuscript in preparation).

Experimental evidence suggests that critical functions of human neutrophils are orchestrated by CD157. Indeed, CD157-mediated signals promote polarization of these cells, an event central to chemotaxis, improving mobility, and becoming instrumental in perceiving the existence of chemotactic gradients (43). When activated neutrophils transform from resting to migratory cells, CD157 localizes into GM1-enriched rafts and migrates preferentially to the uropod. CD157 regulates chemotaxis stimulated through the high affinity fMLP receptor and regulates transendothelial migration (42). Real-time microscopy revealed that CD157 engagement brings a sort of disorientation among neutrophils, which meander toward interendothelial junctions where they eventually stop without transmigrating (43) (Figure 3). These findings are relevant in vivo: indeed CD157-deficient neutrophils obtained from patients with paroxysmal nocturnal hemoglobinuria (who have defective expression of GPI-anchored molecules) are characterized by severely impaired diapedesis and by consistently defective migration.

The relationship between the enzymatic activities and the receptor functions is an intriguing issue, as well as the difference seen between the human and murine models. The molecular details that govern CD157-mediated effects and the hypothesized non-substrate ligand(s) of the molecule remain unknown. Re-

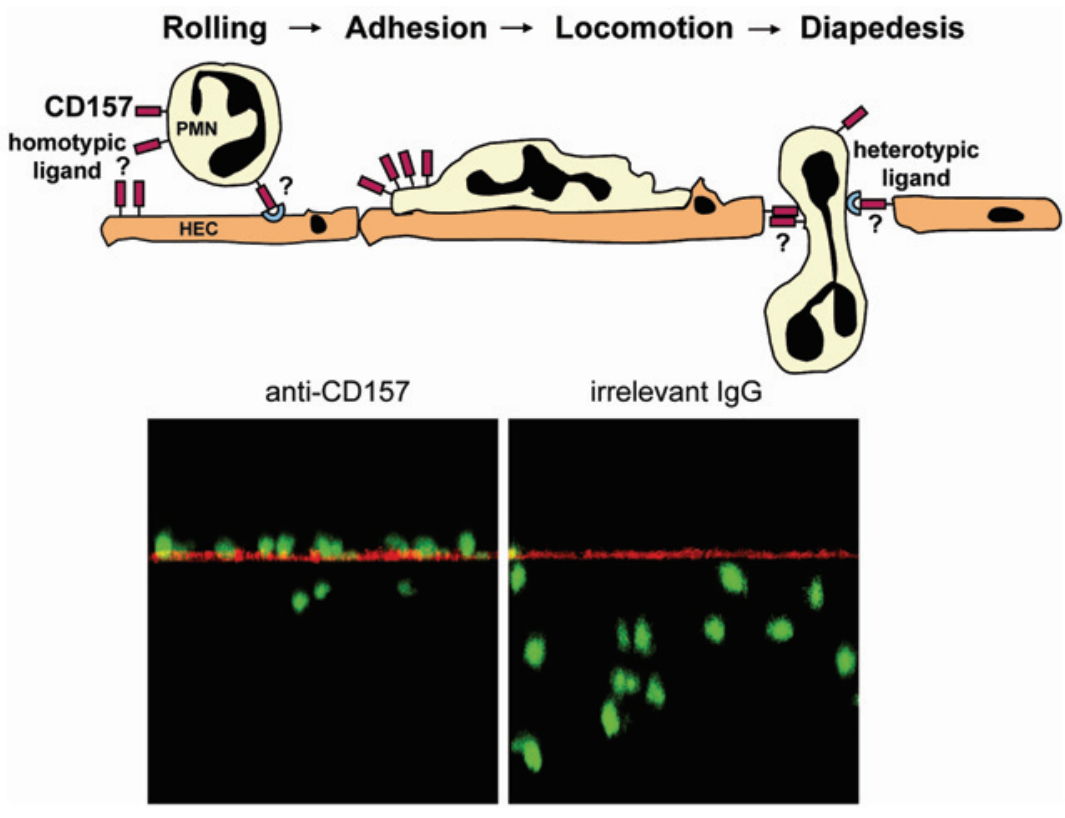

Figure 3. Role of CD157 during neutrophil transendothelial migration: role of CD157. PMN were labeled with carboxyfluorescein succinimidyl ester (CFSE), incubated with anti-CD 157 or irrelevant mAb, and seeded on activated endothelial cell (HEC) monolayers grown on collagen. After migration, samples were fixed, washed, stained with anti-CD31 mAb and F(ab') $)_{2}$-GaMlg-TexasRed and evaluated by laser-scanning confocal microscopy. The images show the position of PMN at the end of the transmigration assay. Samples were analyzed by sequential scanning of the xy planes recorded along the $z$ axis. Series of confocal optical xy images were processed using a 3D-reconstruction program and visualized as orthogonal views. PMN treated with anti-CD $157 \mathrm{mAb}$ show a reduced diapedesis.

cently, Partida-Sanchez et al. (44) demonstrated that CD38 regulates chemotaxis of dendritic cells and dendritic cell precursors during the inflammatory response through the production of cADPR, a $\mathrm{Ca}^{2+}$-mobilizing second messenger. However, it is unlikely that human CD157 could exploit this signal transduction pathway, because its cyclase activity on PMN and endothelial cells is extremely poor (42). Moreover, the enzymatic efficiency of CD157 is several hundredfold lower than that of CD38 and requires acidic $\mathrm{pH}$ and metal ions, far away from a physiological context $(10,11,40,45)$.

\section{CONCLUSIONS}

One of the ambitious aims of the Torino CD38 Meeting was to bring together basic and clinical scientists to highlight the practical aspects of an enormous investment derived from the analysis of ectoenzymes. "No clinic, no success" means that we must address efforts in translating as much as possible our observations in diagnostic and practical applications to warrant a larger visibility and a future for the field, apparently distant from immediate applications. This goal was definitely reached during the Torino Meeting. The issues of localization, access to substrates, transport of products from outside to inside of cells were brilliantly answered and with general agreements. The view of CD38/ CD157 as receptors still has some shadowed areas, partly derived from immunological biases and partly from the different models used (human vs. murine). The results obtained confirm the existence of marked differences be- 
tween murine CD38 and its human counterpart. Results independently obtained from normal and pathological models indicate that CD38 and CD157 act as receptors in man: the effects mediated on CD38 by agonistic mAbs are reproduced using CD31 as counterreceptor or blocked by soluble CD38 and CD31 molecules. A reasonable inference is that CD38 and CD157 may exert more than one function, a concept embraced by the term pleiotropism, a characteristic shared with many other surface molecules, either ectoenzymes or not.

The experience with human models indicates that a high number of functions mediated by CD38 and CD157 are independent of their enzymatic activities. It is reasonable to assume that the large extracellular domains of ectoenzymes and their association with other molecules can mediate response without involvement of their catalytic properties. Further, both molecules are frequently shed from the cell membrane through cleavage or other mechanisms producing soluble forms. This implies that the two molecules might also act as a regulatory system or even bring to interaction with $\mathrm{CD}^{-} 8^{-}$and CD157 cells. High serum concentration of soluble CD157 has been reported in patients with severe rheumatoid arthritis characterized by joint destruction, suggesting a role of molecule in the pathogenesis of the disease (46).

At the end of the Torino Meeting, our impression is that a single model combining the characteristics of enzyme and receptor was not identified. One interpretation is that the two functions are independent and a further possibility is that we are seeing only a few facets of a story and missing some critical keys. The next meeting is requested to view the CD38/CD157 family and-more in general-the ectoenzymes from a physiological view, again using diseases as a way for confirming normality.

\section{ACKNOWLEDGMENTS}

Work supported by grants from Fondazione Internazionale di Ricerca in Medicina Sperimentale (FIRMS), Associ- azione Italiana Ricerca Cancro (AIRC, F.M. and S.D.), PRIN (F.M., A.F. and S.D.), University of Turin (F.M., S.D. and A.F.), Regione Piemonte (F.M.), Chronic Lymphocytic Leukemia Global Research Foundation (CLL-GRF, S.D.), ISS-NIH Scientific Cooperation Agreement (C.M.A.), ISS and AIDS project (C.M.A.), Ministerio de Educación y Ciencia (MEC, Grant SAF2005-06056-C02-01 to J.S.), Consejería de Innovación, Ciencia y Empresa de la Junta de Andalucía (Grant CVI908 to J.S.). M.Z. was supported by the Instituto Carlos III-FIS, Ministerio de Sanidad y Consumo (Grant FIS03/0389) and by a Ramón y Cajal contract from MEC.

Special thanks are given to Compagnia di San Paolo and Fondazione CRT for providing financial support to the CD38 Meeting (www.cd38torino.it).

The help of Drs. K Ishihara and T Hirano (Osaka, Japan) and of Dr. R Villela (Barcelona, Spain) and L Frasca (Rome, Italy) in providing assistance and reagents is gratefully acknowledged. S.A. is on leave of absence from the Hematology Clinics, University of Essen, Essen, Germany and was supported by an ESMO Fellowship.

\section{REFERENCES}

1. States DJ, Walseth TF, Lee HC. (1992) Similarities in amino acid sequences of Aplysia ADP-ribosyl cyclase and human lymphocyte antigen CD38. Trends Biochem. Sci. 17:495.

2. Bricker AL, Carey VJ, Wessels MR. (2005) Role of NADase in virulence in experimental invasive group A streptococcal infection. Infect. Immun. 73:6562-6.

3. Kimoto H, Fujii Y, Yokota Y, Taketo A. (2005) Molecular characterization of NADasestreptolysin O operon of hemolytic streptococci. Biochim. Biophys. Acta. 1681:134-49.

4. Wu Y et al. (1997) Abscisic acid signaling through cyclic ADP-ribose in plants. Science. 278:2126-30.

5. Rusinko N, Lee HC. (1989) Widespread occurrence in animal tissues of an enzyme catalyzing the conversion of NAD+ into a cyclic metabolite with intracellular $\mathrm{Ca}^{+}$-mobilizing activity. J. Biol. Chem. 264:11725-31.

6. Ferrero E, Saccucci F, Malavasi F. (1999) The human CD38 gene: polymorphism, CpG island, and linkage to the CD157 (BST-1) gene. Immunogenetics. 49:597-604.

7. Prasad GS et al. (1996) Crystal structure of Aplysia ADP ribosyl cyclase, a homologue of the bifunctional ectozyme CD38. Nat. Struct. Biol. 3:957-64.
8. Liu Q et al. (2005) Crystal structure of human CD38 extracellular domain. Structure. 13:1331-9.

9. Aturaliya RN et al. (2006) Subcellular localization of mammalian type II membrane proteins. Traffic. 7:613-25.

10. Ziegler M, Niere M. (2004) NAD+ surfaces again. Biochem. J. 382:e5-6.

11. Okamoto H et al. (2000) Physiological and pathological significance of the CD38-cyclic ADP-ribose signaling system. Chem. Immunol. 75:121-45.

12. Okamoto H, Takasawa S. (2002) Recent advances in the Okamoto model: the CD38-cyclic ADPribose signal system and the regenerating gene protein (Reg)-Reg receptor system in $\beta$-cells. Diabetes. 51 Suppl 3:S462-73.

13. Barata $\mathrm{H}$ et al. (2004) The role of cyclic-ADPribose-signaling pathway in oxytocin-induced $\mathrm{Ca} 2+$ transients in human myometrium cells. Endocrinology. 145:881-9.

14. Deshpande DA et al. (2005) CD38/cyclic ADP-ribose signaling: role in the regulation of calcium homeostasis in airway smooth muscle. Am. J. Physiol. Lung Cell. Mol. Physiol. 288:L773-88.

15. Iqbal J, Zaidi M. (2006) Extracellular NAD+ metabolism modulates osteoclastogenesis. Biochem. Biophys. Res. Commun. 349:533-9.

16. Goding JW, Howard MC. (1998) Ecto-enzymes of lymphoid cells. Immunol. Rev. 161:5-10.

17. Hussain AM, Chang CF. (1999) Novel kinetics, behavior and cell-type specificity of CD157mediated tyrosine kinase signaling. Cell. Signal. 11:891-7.

18. Liang F, Qi RZ, Chang CF. (2002) CD157 undergoes ligand-independent dimerization and colocalizes with caveolin in CHO and MCA102 fibroblasts. Cell. Signal. 14:933-9.

19. Zubiaur M, Izquierdo M, Terhorst C, Malavasi F, Sancho J. (1997) CD38 ligation results in activation of the Raf-1/mitogen-activated protein kinase and the CD3-zeta/zeta-associated protein70 signaling pathways in Jurkat $\mathrm{T}$ lymphocytes. J. Immunol. 159:193-205.

20. Morra M, Zubiaur M, Terhorst C, Sancho J, Malavasi F. (1998) CD38 is functionally dependent on the TCR/CD3 complex in human T cells. Faseb. J. 12:581-92.

21. Zubiaur M et al. (2002) CD38 is associated with lipid rafts and upon receptor stimulation leads to Akt/protein kinase B and Erk activation in the absence of the CD3-zeta immune receptor tyrosinebased activation motifs. J. Biol. Chem. 277:13-22.

22. Munoz P et al. (2003) CD38 signaling in T cells is initiated within a subset of membrane rafts containing Lck and the CD3-zeta subunit of the T cell antigen receptor. J. Biol. Chem. 278:50791-802.

23. Drevot P et al. (2002) TCR signal initiation machinery is pre-assembled and activated in a subset of membrane rafts. Embo. J. 21:1899-1908.

24. Schade AE, Levine AD. (2002) Lipid raft heterogeneity in human peripheral blood $\mathrm{T}$ lymphoblasts: a mechanism for regulating the initiation of TCR signal transduction. J. Immunol. 168: 2233-9. 
25. Slaughter $\mathrm{N}$ et al. (2003) The flotillins are integral membrane proteins in lipid rafts that contain TCR-associated signaling components: implications for T-cell activation. Clin. Immunol. 108: 138-51.

26. Pavon EJ et al. (2006) Increased association of CD38 with lipid rafts in T cells from patients with systemic lupus erythematosus and in activated normal T cells. Mol. Immunol. 43:1029-39.

27. Blanco P, Palucka AK, Gill M, Pascual V, Banchereau J. (2001) Induction of dendritic cell differentiation by IFN- $\alpha$ in systemic lupus erythematosus. Science. 294:1540-3.

28. Deaglio S, Vaisitti T, Aydin S, Ferrero E, Malavasi F. (2006) In-tandem insight from basic science combined with clinical research: CD38 as both marker and key component of the pathogenetic network underlying chronic lymphocytic leukemia. Blood. 108:1135-44.

29. Matrai Z. (2005) CD38 as a prognostic marker in CLL. Hematology 10:39-46.

30. Deaglio S et al. (2003) CD38 is a signaling molecule in B-cell chronic lymphocytic leukemia cells. Blood. 102:2146-55.

31. Deaglio S et al. (2005) CD38 and CD100 lead a network of surface receptors relaying positive signals for B-CLL growth and survival. Blood. 105:3042-50.

32. Randolph GJ, Sanchez-Schmitz G, Angeli V. (2005) Factors and signals that govern the migration of dendritic cells via lymphatics: recent advances. Springer Semin. Immunopathol. 26:273-87.

33. Fedele G et al. (2004) CD38 is expressed on human mature monocyte-derived dendritic cells and is functionally involved in CD83 expression and IL-12 induction. Eur. J. Immunol. 34:1342-50.

34. Deaglio S, Malavasi F. (2006) The CD38/CD157 mammalian gene family: an evolutionary paradigm for other leukocyte surface enzymes. Purinergi Signaling. 2:431-41.

35. Frasca L et al. (2006) CD38 orchestrates migration, survival, and Th1 immune response of human mature dendritic cells. Blood. 107:2392-9.

36. Harrington LE, Mangan PR, Weaver CT. (2006) Expanding the effector CD4 T-cell repertoire: the Th17 lineage. Curr. Opin. Immunol. 18:349-56.

37. Itoh M et al. (1994) Molecular cloning of murine BST-1 having homology with CD38 and Aplysia ADP-ribosyl cyclase. Biochem. Biophys. Res. Commun. 203:1309-17.

38. Kaisho T et al. (1994) BST-1, a surface molecule of bone marrow stromal cell lines that facilitates pre-B-cell growth. Proc. Natl. Acad. Sci. U. S. A. 91:5325-9.

39. Okuyama Y et al. (1996) Human BST-1 expressed on myeloid cells functions as a receptor molecule. Biochem. Biophys. Res. Commun. 228:838-45.

40. Hussain AM, Lee HC, Chang CF. (1998) Functional expression of secreted mouse BST-1 in yeast. Protein Expr. Purif. 12:133-7.

41. Ishihara K, Hirano T. (2000) BST-1/CD157 regulates the humoral immune responses in vivo. Chem. Immunol. 75:235-55.
42. Funaro A et al. (2004) CD157 is an important mediator of neutrophil adhesion and migration. Blood. 104:4269-78.

43. Ortolan E et al. (2006) CD157 plays a pivotal role in neutrophil transendothelial migration. Blood. 108:4214-22.

44. Partida-Sanchez S et al. (2004) Chemotaxis and calcium responses of phagocytes to formyl peptide receptor ligands is differentially regulated by cyclic ADP ribose. J. Immunol. 172:1896-1906.

45. Hirata Y et al. (1994) ADP ribosyl cyclase activity of a novel bone marrow stromal cell surface molecule, BST-1. FEBS Lett. 356:244-8.

46. Lee BO et al. (1996) Elevated levels of the soluble form of bone marrow stromal cell antigen 1 in the sera of patients with severe rheumatoid arthritis. Arthritis. Rheum. 39:629-37. 